\title{
Automated Mammographic Reporting and Follow-up With an Inexpensive Lap-Top Microcomputer
}

\author{
Morgan G. Dunne and Sean B. Dunne
}

This report describes the use of an inexpensive, lap-top microcomputer and simple, user-friendly BASIC program for automated mammographic reporting. The system affords data-base filing of those cases wherein surgical and/or mammographic followup is indicated.

(c) 1989 by W.B. Saunders Company.

KEY WORDS: mammography, reporting, computers, radiology, software, breast cancer.

$\mathbf{I}^{\mathrm{N}}$ N AN EFFORT TO EXPEDITE, standardize, and follow up on mammographic reports, a simple BASIC program was developed for use on an inexpensive lap-top microcomputer. Automated mammographic systems have been described in the literature, but these may require expensive computer hardware and software or a terminal linked to a central hospital computer system with appropriate software available on line. ${ }^{1,2,3}$ The system described herein allows the practicing radiologist to generate printed reports rapidly on mammographic images, without the need for a transcriptionist or dictating system, using a very inexpensive lap-top computer and electronic typewriter. The program was designed to minimize the number of keystrokes necessary to generate a printed report and allow ease of use by those lacking typing skills or those unfamiliar with computers. An enhancement of the program includes a simple data-base file, allowing the radiologist to maintain an upgradable file on those cases in which surgical or mammographic follow-up is indicated. By this means, interval printouts can be obtained listing patients in whom biopsies and/or additional mammographic examinations were recommended; thus, reminders can be forwarded to the referring physicians and/or patients themselves.

The program was written in BASIC on a Radio Shack TRS-80 Model 100/102 portable lap-top computer (Tandy Corporation, Fort Worth, TX). BASIC is probably the language most familiar to nonprofessional computer programmers, and the use of this language allows individual users to modify the program code and thus customize the program to their needs. The lap-top computer selected has a permanent, nonvolatile random access memory (RAM), which comes with the BASIC language and text editor installed. The unit contains a built-in clock/ calendar, which maintains the day, date and time in permanent RAM.

The program operates almost exclusively by the use of only two keys, the space bar and Return, thus obviating the need for significant typing on the part of the user. Only if users select an interpretation that necessitates follow-up will they be asked to enter string data such as patient name and identification number. The program is designed to be used with an electronic typewriter that is connected to the lap-top computer so that the finished report is printed directly onto the mammogram request form, which already contains the patient's identification, clinical and demographic data. The program can be modified to allow the report to be printed on continuous computer paper, but then the patient data must be entered for every case. The date and time of the interpretation and name of the radiologist are automatically included on every printed report.

The program operates by offering serial choices on the screen, which the user selects by pressing the Return key or the space bar. Initially the program will ask whether the study is a xeromammogram or film-screen mammogram, and whether or not a prior study is available for comparison. The program will then ask the user if a standard negative report or an itemized report is to be prepared. The program contains a standard negative report that has been individually designed by the user to cover all unremarkable or negative examinations. Such a negative report is shown in Table 1. Experience shows that the negative report covers most studies in a busy mammographic service, especially where large scale screening is performed. Thus, most cases can be reported without the need for any further keyboard entries by the user.

From the Department of Radiology, Scott \& White, Temple, $T X$.

Address reprint requests to Morgan G. Dunne, $M B, B C h$, Department of Radiology, Scott \& White, 2401 South 31st St, Temple, $T X 76508$.

1989 by W.B. Saunders Company.

0897-1889/89/0203-0004\$03.00/0 
Table 1. Standard Negative Report

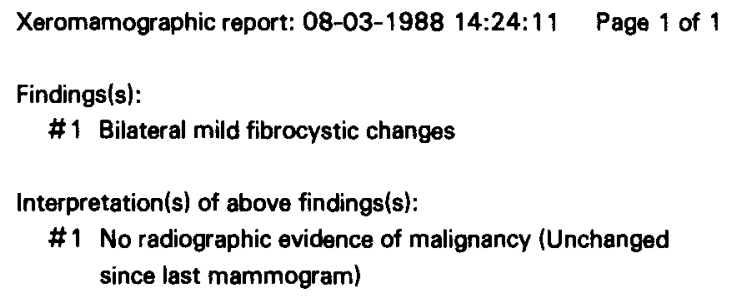

If the user opts for an itemized report, the screen will serially display a list of findings (Table 2), which are either accepted or rejected by the user. Once a finding is selected, a series of adjectives pertinent to that finding are displayed, and one of these is then selected. Next, a series of breast locations are offered, and the appropriate selection is made. As the selection process proceeds, the report is built on the top half of the screen, and the offered choices are displayed at the bottom of the screen, one at a time. Subsequently, the user is presented with a series of interpretations (Table 3 ) referable to the finding described, and a selection is made. If the user had stated at the beginning of the run that a prior mammogram was available, and when the description of the finding and its location and interpretation are complete, the user is offered a series of phrases indicating whether or not the finding has developed or changed in the interim. At this point, the user opts to print the report or add additional findings, making as many iterations through the finding subroutine as necessary. A sample itemized report is shown in Table 4.

If the user selects an interpretation that requires follow-up, such as "ABNORMAL MAM-

Table 2. Mammographic Findings

Fibocystic changes

Fatty replacement

Calcifications

Microcalcifications

Asymmetric density

Well-defined mass

III-defined mass

Surgical scars

Skin changes

Visible lymph nodes

Dilated retroareolar duct

Nipple retraction

Mammary prosthesis
Table 3. Mammographic Interpretations

No radiographic evidence of malignancy

Normal involutional appearance

Probable benign cyst of fibroadenoma

ABNORMAL MAMMOGRAM, REPEAT IN FOUR MONTHS

SUSPICIOUS FOR CANCER: SUGGEST BIOPSY

Skin mole

Intrammary lymph node

MOGRAM, REPEAT IN FOUR MONTHS" or "SUSPICIOUS FOR CANCER: SUGGEST BIOPSY," the program will request that the patient's name, identification number, and referring physician's name be entered. The program will then place the entered data, current date, and matching report into a simple data-base file in the permanent RAM of the computer. This file can subsequently be viewed, edited, and printed out to allow the user to keep track of those patients in need of follow-up. The follow-up file is an important feature of the system with significant quality assurance and medicolegal advantages. ${ }^{4,5}$ Use of the built-in text editor and file-management software in the computer allows the file to be searched for a particular record, data to be added, or for deletion of the record once appropriate follow-up care has been rendered.

The inclusion of multiple findings and interpretations may make the printed report run to two or more pages, depending on the size of the radiology request form at a given institution or office practice. The maximum report form length is embedded in the program, and printing is halted to allow the user to insert a blank request form

Table 4. Itemized Mammographic Report

Xeromammogram report: 08-03-1988 14:28:52 Page 1 of 1

Finding(s):

\# 1 Bilateral mild fatty replacement

\#2 Right breast punctate microcalcifications superior lateral quadrant at 9 o'clock

\#3 Left breast 5 to $10 \mathrm{~mm}$ well defined mass inferior medical quadrant at 8 o' clock

Interpretation(s) of above findings(s):

\# 1 Normal involutional appearance (Unchanged since last mammogram)

\#2 SUSPICIOUS FOR CANCER: SUGGEST BIOPSY (Developed since last mammogram)

\#3 Probable benign cyst or fibroadenoma (Unchanged since last mammogram)

J. Smith, M.D./mm 
into the electronic typewriter for each subsequent page. If the report in a given case runs to more than one page, a random number paging code generated by the program is placed on each printed page, to allow the user to match the printed pages to a specific patient and ensure that different patients' multiple page reports do not become mixed.

The program takes up less than 7 kilobytes
(kB) of RAM of the 24 or $32 \mathrm{kB}$ RAM that comes already installed in the typical 24 or $32 \mathrm{kB}$ model $100 / 102$ computer (an additional $2.6 \mathrm{kB}$ is taken up by BASIC). The permanent nonvolatile RAM can be expanded with the addition of memory chips as desired by the user. It takes less than 30 seconds to generate a standard "normal" report and less than one minute for an itemized report with three findings.

\section{REFERENCES}

1. Haug PJ, Tocino IM, Clayton PD, et al: Automated management of screening and diagnostic mammography. Radiology 164:747-752, 1987

2. Pryor TA, Gardner RM, Clayton PD, et al: The HELP system. J Med Sys 7:87-102, 1983

3. Bates BF: A personal-computer reporting system for mammography. AJR 151:1237-1239, 1988

4. Brenner RJ: Medical legal aspects of a screening mammography: A primer. Represented at the categorical course on breast imaging at the 88th annual meeting of the American Roentgen Ray Society, San Francisco, CA, May 1988.

5. McLelland R: How to establish a low cost community screening program for breast cancer. Presented at the categorical course on breast imaging at the 88th annual meeting of the American Roentgen Ray Society, San Francisco, CA, May 1988. 